\title{
MANIPULACIÓN DE LESIONES EN PACIENTES CON LEISHMANIASIS CUTÁNEA: SERIE DE CASOS EN UN HOSPITAL PERUANO
}

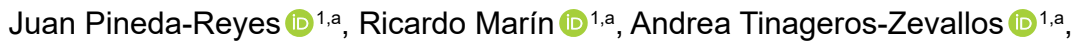 \\ Ana P. Ramos (1) 2,a, Fiorela Alvarez $\mathbb{1}^{2, b}$, Alejandro Llanos-Cuentas $\mathbb{1}^{2, b, c}$ \\ 1 Facultad de Medicina Alberto Hurtado, Universidad Peruana Cayetano Heredia, Lima, Perú. \\ ${ }^{2}$ Instituto de Medicina Tropical Alexander von Humboldt, Universidad Peruana Cayetano Heredia, Lima, Perú. \\ ${ }^{a}$ Médico cirujano; ${ }^{b}$ médico especialista en Enfermedades infecciosas y tropicales; ${ }^{c}$ doctor en Medicina y \\ Epidemiología. \\ El presente estudio forma parte de la tesis: Pineda-Reyes J, Marín R, Tinageros-Zevallos A. El efecto de la \\ manipulación de lesiones de pacientes con leishmaniasis cutánea en una serie hospitalaria de un centro de referencia \\ nacional durante el período junio 2017 - diciembre 2017 [tesis de bachiller]. Lima: Facultad de Medicina Alberto \\ Hurtado, Universidad Peruana Cayetano Heredia; 2019
}

\section{RESUMEN}

En áreas endémicas de leishmaniasis cutánea es común que los pacientes manipulen sus lesiones con tratamientos tradicionales como primera opción terapéutica. Con el objetivo de describir la frecuencia y los cambios de la manipulación de lesiones de leishmaniasis cutánea de pacientes en el Hospital Cayetano Heredia, se realizó un estudio tipo serie de casos. Se incluyeron 124 pacientes con leishmaniasis cutánea. El 54\% (67/124) manipuló sus lesiones; el 92,5\% (62/67) lo hizo con productos químicos, y el $43,3 \%$ (29/67), con plantas. Los cambios locales más frecuentemente reportados por los pacientes fueron aumento de tamaño de la lesión en el 35,8\% (24/67) e incremento de inflamación en el 28,4\% (19/67). La manipulación disminuyó la positividad del diagnóstico parasitológico en aquellos pacientes con lesiones ulcerativas.

Palabras clave: Leishmaniasis cutánea; Medicina Tradicional; Diagnóstico (fuente: DeCS BIREME).

\section{MANIPULATION OF LESIONS IN PATIENTS WITH CUTANEOUS LEISHMANIASIS: CASE SERIES IN A PERUVIAN HOSPITAL}

\begin{abstract}
In cutaneous leishmaniasis endemic areas it is a common practice for patients to manipulate their lesions with traditional treatments as a first therapeutic option. A case series study was conducted in order to describe the frequency and the variations of the patient manipulation of cutaneous leishmaniasis lesions at the Cayetano Heredia Hospital. The study included 124 patients with cutaneous leishmaniasis. From the patient population it was found that 54\% (67/124) manipulated their lesions. Of this, $92.5 \%$ (62/67) did so with chemicals, and 43.3\% (29/67) with plants. The most frequent local changes reported by patients were increased lesion size in $35.8 \%$ (24/67) and increased inflammation in $28.4 \%(19 / 67)$. Manipulation by patients decreased the positivity of the parasitological diagnosis in those patients with ulcerative lesions.
\end{abstract}

Citar como: Pineda-Reyes J, Marín R, Tinageros-Zevallos A, Ramos AP, Alvarez F, Llanos-Cuentas A. Manipulación de lesiones en pacientes con leishmaniasis cutánea: serie de casos en un hospital peruano. Rev Peru Med Exp Salud Publica. 2020;37(2):265-9. doi: https://doi. org/10.17843/rpmesp.2020.372.4799

Correspondencia: Alejandro Llanos Cuentas; Av. Honorio Delgado 262, San Martín de Porres, Lima, Perú; alejandro.llanos.c@upch.pe

Recibido: $12 / 09 / 2019$

Aprobado: 13/04/2020

En línea: 10/06/2020
Keywords: Leishmaniasis, Cutaneous; Medicine, Traditional; Diagnosis (source: MeSH NLM).

\section{INTRODUCCIÓN}

La leishmaniasis es una enfermedad desatendida causada por protozoarios del género Leishmania adquiridos por la picadura de insectos del género Lutzomyia ${ }^{(1)}$. La leishmaniasis cutánea (LC) es la forma clínica más común y en el Perú se reportan aproximadamente 7000 casos cada año ${ }^{(1-4)}$.

En zonas endémicas, particularmente en áreas rurales, es frecuente el uso de tratamientos tradicionales como primera opción, debido a la falta de acceso a servicios de salud, desconfianza en la medicina occidental, poca disponibilidad del tratamiento estandarizado, temor a efectos secundarios, entre otros ${ }^{(3,5)}$. Los tratamientos tradicionales incluyen la aplicación tópica de plantas, sustancias químicas o térmicas, antibióticos, etc. ${ }^{(3,5-7)}$ El uso de estos productos 
podría alterar la morfología de la lesión y disminuir su carga parasitaria, alterar la histopatología y, en consecuencia, el diagnóstico de la enfermedad. Esto cobra importancia en el Perú pues se tiene como requisito la demostración parasitológica para el inicio de tratamiento estandarizado gratuito ${ }^{\left({ }^{8}\right)}$. Cabe resaltar, que actualmente existen otros métodos de diagnóstico de LC, como el cultivo, la histopatología y métodos moleculares, siendo estos menos accesibles en nuestro medio.

A la fecha, se desconoce la prevalencia de la manipulación de lesiones en hospitales de referencia o cómo esta práctica afectaría el diagnóstico por microscopía óptica y la apariencia de la lesión. Este estudio describe la frecuencia y los tipos de manipulación, en el contexto del resultado de microscopía óptica (frotis) y las características clínicas de lesiones de LC.

\section{EL ESTUDIO}

Se realizó un estudio observacional descriptivo, tipo serie de casos, donde se analizó la información obtenida de los registros clínicos del Programa de Leishmaniasis del Instituto de Medicina Tropical Alexander von Humboldt de la Universidad Peruana Cayetano Heredia, un centro de referencia en Lima que diagnosticó y trató a pacientes con leishmaniasis de junio a diciembre de 2017.

Los médicos del Programa de Leishmaniasis consideraron como casos a los registros de pacientes de todas las edades con diagnóstico establecido de LC no complicada (sin compromiso mucoso y en huésped inmunocompetente). Se evaluaron criterios epidemiológicos, clínicos, parasitológicos o inmunológicos. El diagnóstico clínico estuvo basado en lo siguiente: presencia de úlcera, bordes regulares, bien definidos, indurados, violáceos, elevados en rodete, con fondo granulomatoso y grueso ${ }^{(9)}$. Se incluyeron registros que contaron con resultados de frotis y prueba cutánea de leishmanina (LST, por sus siglas en inglés), siendo al menos uno de estos positivo, y se excluyeron registros con datos incompletos.

La manipulación de lesiones se definió como la aplicación tópica de plantas en al menos dos oportunidades (colocación directa, emplastos, infusión), sustancias químicas (ácido de batería, piedra azul o productos químicos farmacéuticos como cremas antibióticas, antimicóticas o corticoides) o térmicas. Fueron los propios pacientes, a excepción de los menores de edad, quienes manipularon sus lesiones. Se describió el cambio que dicha manipulación tuvo sobre las lesiones según lo informado por los pacientes en los registros clínicos. Dicho cambio se clasificó en aumento/ disminución de tamaño de la lesión (diámetro), aumento/ disminución de la inflamación (eritema, dolor, edema, secreciones) o ninguno.

Los datos fueron ingresados a una base de datos creada en Excel ${ }^{\circ}$. Para el análisis de la información, se utilizó Stata 15 (StataCorp, College Station, Texas, EUA) y StatCalc en

\section{MENSAJES CLAVE}

Motivación para realizar el estudio: En la actualidad, se desconoce la frecuencia de la manipulación de heridas por leishmaniasis cutánea (LC) en hospitales y su repercusión en el diagnóstico por microscopía.

Principales hallazgos: Más de la mitad de pacientes con LC manipuló sus lesiones, la mayoría con productos químicos. Hubo un aumento del tamaño de las lesiones luego de su manipulación. En los pacientes con úlceras, la manipulación obtuvo resultados negativos en la microscopía.

Implicancias: El diagnóstico de leishmaniasis por microscopía es muy utilizado en el primer nivel de atención. Por ello, se debe educar al paciente para disminuir la manipulación de las lesiones, generar un diagnóstico precoz y un tratamiento oportuno.

EpiInfo $^{\text {tw }}$. Se calcularon frecuencias, proporciones, medidas de tendencia central y dispersión. Se cruzaron las variables cualitativas de microscopía, sexo, tiempo de enfermedad, grupo etario y tipo de lesión con la presencia de manipulación y el resultado del frotis. Se determinó su significancia estadística con las pruebas de chi cuadrado y exacta de Fisher.

El proyecto fue aprobado por los comités de ética de la Universidad Peruana Cayetano Heredia y del Hospital Cayetano Heredia (constancia 0670519).

\section{HALLAZGOS}

Desde junio hasta diciembre de 2017, se identificaron 150 pacientes con LC, 26 de los cuales fueron excluidos por información incompleta; así, el total de pacientes fue 124. La razón de hombres/mujeres fue 2,64. La mediana de edad fue 38 años (rango intercuartil 22-50) y el grupo etario predominante fue de adultos en $71,8 \%$ (89/124) (Tabla 1). El $68,6 \%(85 / 124)$ de pacientes procedía del departamento de Lima. Respecto al lugar de contagio destacaron Lima, Madre de Dios y Cusco.

La mayoría de pacientes presentó una lesión $(59,7 \%$ [74/124]) y el 16,9\%, tres o más. Se registró un total de 205 lesiones. La lesión más común fue úlcera en el 72,7\% (149/205) de las lesiones (Tabla 1), seguida de lesiones nodulares e infiltrativas. Las localizaciones más frecuentes fueron miembro superior, con 32,7\% (67/205) de las lesiones, y cabeza y miembro inferior, ambos con 27,8\% (57/205) de las lesiones.

El frotis fue positivo en 73,4\% (91/124) de los pacientes y la LST en el 91,9\% (114/124) (Tabla 1). Analizando todos los tipos de lesiones, no se observó diferencia estadística $(\mathrm{p}=0,198)$ en la positividad del frotis entre las lesiones manipuladas $(50,5 \%)$ y no manipuladas $(49,5 \%)$. Sin embargo, 
Tabla 1. Características clínicas y de laboratorio de 124 pacientes con leishmaniasis cutánea y manipulación de lesiones que fueron atendidos en el Instituto de Medicina Tropical de la Universidad Peruana Cayetano Heredia.

\begin{tabular}{|c|c|c|c|}
\hline Característica & $\begin{array}{c}\text { Manipulados } \\
\mathbf{n}(\%)\end{array}$ & $\begin{array}{c}\text { No } \\
\text { manipulados } \\
\mathbf{n}(\%) \\
\end{array}$ & $\begin{array}{l}\text { Valor } \\
\text { de } p^{\text {b }}\end{array}$ \\
\hline Sexo & & & 0,800 \\
\hline Masculino & $48(53,7)$ & $42(46,7)$ & \\
\hline Femenino & $19(55,9)$ & $15(44,1)$ & \\
\hline Procedencia & & & 0,678 \\
\hline Lima & $47(55,3)$ & $38(44,7)$ & \\
\hline Provincia & $20(51,3)$ & $19(48,7)$ & \\
\hline Frotis & & & 0,198 \\
\hline Positivo & $46(50,5)$ & $45(49,5)$ & \\
\hline Negativo & $21(63,6)$ & $12(36,4)$ & \\
\hline $\begin{array}{l}\text { Frotis de pacientes con } \\
\text { úlceras }(\mathrm{n}=100)\end{array}$ & & & 0,029 \\
\hline Positivo & $35(46,7)$ & $40(53,3)$ & \\
\hline Negativo & $18(72,0)$ & $7(28,0)$ & \\
\hline Tiempo de enfermedad ${ }^{\mathrm{a}}$ & & & 0,111 \\
\hline$\leq 3$ meses & $28(46,7)$ & $32(53,3)$ & \\
\hline$>3$ meses & $39(60,9)$ & $25(39,1)$ & \\
\hline \multicolumn{4}{|l|}{ Grupo etario $^{\mathrm{a}}$} \\
\hline Niño & $8(53,3)$ & $7(46,7)$ & 0,954 \\
\hline Adolescente & $5(62,5)$ & $3(37,5)$ & $0,725^{\mathrm{c}}$ \\
\hline Adulto & $49(55,1)$ & $40(44,9)$ & 0,716 \\
\hline Adulto mayor & $5(41,7)$ & $7(58,3)$ & 0,382 \\
\hline \multicolumn{4}{|l|}{ Tipo de lesión $(\mathrm{n}=205)^{\mathrm{a}}$} \\
\hline Ulcerosa & $71(47,7)$ & $78(52,3)$ & 0,059 \\
\hline Nodular & $18(62,1)$ & $11(37,9)$ & 0,229 \\
\hline Infiltrativa & $6(54,5)$ & $5(45,5)$ & 1,000 \\
\hline Verrucosa & $2(100)$ & $0(0,0)$ & $0,498^{c}$ \\
\hline Costrosa & $0(0,0)$ & $2(100)$ & $0,232^{\mathrm{c}}$ \\
\hline Polimórfica & $9(75,0)$ & $3(25,0)$ & $0,137^{\mathrm{c}}$ \\
\hline
\end{tabular}

a Valor de $p$ calculado comparando la proporción con el complemento de la categoría

b Prueba de chi cuadrado

${ }^{c}$ Prueba exacta de Fisher

considerando únicamente las lesiones ulcerativas, la manipulación redujo significativamente la positividad del frotis $(p=0,029)$. Sesenta y siete pacientes $(54,0 \%)$ manipularon sus lesiones, tanto adultos como niños, lo cual representa el $65,7 \%(44 / 67)$ y el $16,4 \%(11 / 67)$, respectivamente. Los pacientes que manipularon sus lesiones procedían principalmente de Lima (Tabla 1).

En las lesiones de mayor tiempo de enfermedad (más de tres meses), la demostración parasitológica fue significativamente menor independientemente de la ocurrencia de manipulación (Tabla 2). Los productos más utilizados fueron los químicos en el 92,5\% (62/67) de los casos, y las plantas, en el 43,3\% (29/67). El 49,3\% (33/67) de los pacientes usó solo químicos y apenas el 6,0\% (4/67), únicamente plantas, mientras que el 34,3\% (23/67) reportó haber utilizado ambos productos.
Tabla 2. Resultado de frotis según manipulación y tiempo de enfermedad en pacientes portadores de leishmaniasis cutánea que fueron atendidos en el Instituto de Medicina Tropical de la Universidad Peruana Cayetano Heredia.

\begin{tabular}{lccc}
\hline \multirow{2}{*}{ Manipulación } & \multicolumn{2}{c}{ Tiempo de enfermedad } & Valor de $\mathbf{p}$ \\
\cline { 2 - 4 } & $\leq \mathbf{3}$ meses & $>\mathbf{3}$ meses & \\
\hline Manipulados & & & $0,003^{\mathrm{a}}$ \\
Frotis positivo & 25 & 21 & \\
Frotis negativo & 3 & 18 & \\
No manipulados & & & $0,003^{\mathrm{a}}$ \\
$\quad$ Frotis positivo & 30 & 15 & \\
Frotis negativo & 2 & 10 & \\
\hline
\end{tabular}

a Prueba exacta de Fisher

Sesenta y dos pacientes emplearon químicos para manipular sus lesiones; los más utilizados fueron la crema antibiótica o antimicótica y el agua oxigenada (Tabla 3). Asimismo, los pacientes informaron haber usado las siguientes plantas: Plantago major (llantén), Piper aduncum (matico), Chamaemelum nobile (manzanilla), Oenothera rosea (chupasangre), entre otras. Veintinueve pacientes manipularon sus lesiones con plantas, siendo el llantén la más empleada, seguido del matico y la manzanilla. Seis pacientes emplearon quemaduras térmicas y otros seis utilizaron productos como hígado de res, grasa de cerdo, miel, orina y excretas.

Finalmente, el cambio más reportado fue el aumento del tamaño con 35,8\% (24/67), seguido por el aumento de la inflamación con $28,4 \%$ (19/67). El 31,3\% (21/67) no reportó cambios en las lesiones (Tabla 4).

Tabla 3. Productos usados en lesiones de leishmaniasis cutánea por pacientes atendidos en el Instituto de Medicina Tropical de la Universidad Peruana Cayetano Heredia $(\mathrm{n}=67)$

\begin{tabular}{lr}
\hline Producto & n (\%) \\
\hline Químicos & $62(92,5)$ \\
Crema antibiótica & $16(23,9)$ \\
Agua oxigenada & $14(20,9)$ \\
Crema antimicótica & $9(13,4)$ \\
Tableta antibiótica & $7(10,5)$ \\
Corticoide tópico & $5(7,5)$ \\
Limón & $4(6,0)$ \\
Sal & $4(6,0)$ \\
«Piedra azul» & $3(4,5)$ \\
Ácido de batería & $3(4,5)$ \\
Violeta de genciana & $3(4,5)$ \\
Otros & $37(55,2)$ \\
Plantas & $29(43,3)$ \\
Plantago major (llantén) & $13(19,4)$ \\
Piper aduncum (matico) & $8(11,9)$ \\
Chamaemelum nobile (manzanilla) & $5(7,5)$ \\
Oenothera rosea (chupasangre) & $3(4,5)$ \\
Croton lechleri (sangre de grado) & $3(4,5)$ \\
Otros & $12(17,9)$ \\
\hline
\end{tabular}


Tabla 4. Cambios observados en lesiones manipuladas de leishmaniasis cutánea por pacientes atendidos en el Instituto de Medicina Tropical de la Universidad Peruana Cayetano Heredia $(n=67)$

\begin{tabular}{|c|c|}
\hline Característica & n (\%) \\
\hline \multicolumn{2}{|l|}{ Tipo de cambio } \\
\hline Aumento del tamaño & $24(35,8)$ \\
\hline Aumento de la inflamación & $19(28,4)$ \\
\hline Disminución de la inflamación & $7(10,5)$ \\
\hline Disminución del tamaño & $2(3,0)$ \\
\hline Ningún cambio & $21(31,3)$ \\
\hline \multicolumn{2}{|l|}{ Cambios más frecuentes } \\
\hline \multicolumn{2}{|l|}{ Solo plantas } \\
\hline Ningún cambio & $2(3,0)$ \\
\hline Aumento del tamaño & $1(1.5)$ \\
\hline Aumento de la inflamación & $1(1.5)$ \\
\hline \multicolumn{2}{|l|}{ Solo sustancias químicas } \\
\hline Aumento del tamaño & $15(22.4)$ \\
\hline Aumento de la inflamación & $10(14,9)$ \\
\hline Ningún cambio & $9(13,4)$ \\
\hline Disminución de la inflamación & $2(3,0)$ \\
\hline Disminución del tamaño & $1(1,5)$ \\
\hline \multicolumn{2}{|l|}{ Solo térmicos } \\
\hline Aumento del tamaño & $1(1,5)$ \\
\hline \multicolumn{2}{|l|}{ Plantas y sustancias químicas } \\
\hline Ningún cambio & $10(14,9)$ \\
\hline Aumento de la inflamación & $6(9,0)$ \\
\hline Aumento del tamaño & $5(7,5)$ \\
\hline Disminución de la inflamación & $3(4,5)$ \\
\hline \multicolumn{2}{|l|}{ Químicos y térmicos } \\
\hline Disminución de la inflamación & $1(1,5)$ \\
\hline
\end{tabular}

\section{DISCUSIÓN}

En esta serie de casos, la manipulación de lesiones ulcerativas de LC con tratamientos tradicionales redujo significativamente la demostración parasitológica. Esta práctica impide el diagnóstico definitivo, necesario para que un paciente con LC reciba tratamiento gratuito, según lo especifica la norma nacional de tratamiento de leishmaniasis del Ministerio de Salud ${ }^{(8)}$.

Más de la mitad de los pacientes con LC (54,0\%) llegaron a la consulta con antecedente de haber manipulado sus lesiones; la mayoría de ellos con múltiples productos, generalmente químicos (92\%). Sin embargo, la literatura muestra frecuencias de manipulación mayores $(71,2 \%)$ en poblaciones de zonas endémicas de leishmaniasis de larga data ${ }^{(3)} y$ en estudios de países latinoamericanos ${ }^{(10-12)}$. En poblaciones rurales de zonas endémicas de LC es común usar medicina tradicional como tratamiento, incluso en comunidades con servicios de salud ${ }^{(3)}$. El origen de esta cultura se atribuye a la falta de información o percepciones distorsionadas sobre la enfermedad, el deseo de impedir su progresión o la falta de un diagnóstico adecuado ${ }^{(10,12)}$.

La mayoría de los pacientes del estudio eran varones, probablemente debido a que ellos tienen un mayor riesgo de transmisión ${ }^{(13,14)}$. Se han informado hallazgos similares en Guatemala y en Ecuador ${ }^{(10,12)}$, donde los asocian al riesgo ocupacional (agricultura, ganadería). No obstante, hay áreas donde los menores de 15 años son los más vulnerables, sobre todo cuando la transmisión ocurre dentro del domicilio ${ }^{(13)}$.

La manipulación de las lesiones frecuentemente ocurre antes que los afectados busquen un centro de salud ${ }^{(3,12)}$, lo cual afecta las opciones diagnósticas negativamente. No solo disminuye la probabilidad de detectar el parásito con el frotis, sino que también altera la morfología de las lesiones, dificultando el diagnóstico clínico por personal de salud con limitada capacitación. Basado en la experiencia del Hospital Cayetano Heredia, es relativamente frecuente que cualquier úlcera crónica en pacientes de zonas rurales sea catalogada como leishmaniasis, correspondiendo en muchos casos a otras enfermedades como esporotricosis, infecciones piógenas, picaduras de insectos, cáncer de piel, tuberculosis cutánea, entre otras. Asimismo, la manipulación de las lesiones retrasa y encarece el diagnóstico de la LC. Lo usual en el Hospital Cayetano Heredia es que para las lesiones no manipuladas se obtenga el resultado del frotis en pocas horas y se inicie el tratamiento al día siguiente. Sin embargo, en pacientes con lesiones manipuladas, el diagnóstico supera la semana y se requieren exámenes adicionales, como cultivos, anatomía patológica o reacción en cadena de la polimerasa (PCR, por sus siglas en inglés). Esto implica el incremento de costos para los pacientes (prolongación de su estadía en Lima) y para el sistema de salud.

Se conoce que el tiempo de enfermedad afecta el diagnóstico parasitológico, especialmente los métodos de diagnóstico clásicos ${ }^{(15,16)}$. En este estudio, se encontró que las lesiones con mayor tiempo de enfermedad (tres meses a más), independientemente de la presencia de manipulación, tuvieron menor proporción de frotis positivo, debido a la reducción de la carga parasitaria a consecuencia de la respuesta inmune ${ }^{(15)}$.

Los pobladores de comunidades endémicas manipulan sus lesiones principalmente con productos cáusticos; así la gran mayoría empleó sustancias químicas (92\%) y menos de la mitad (43\%) usó plantas. Al menos un tercio de los que manipularon sus lesiones reconocieron que hubo aumento del tamaño de la lesión o inflamación. La manipulación de lesiones frecuentemente produce inflamación, sobreinfección, necrosis ${ }^{(3)}$ y quemaduras de primer a tercer grado ${ }^{(12)}$. La intensidad del daño depende del producto utilizado; el uso de azufre o ácidos generan más daño, lo que provoca celulitis extensas. Las plantas causaron menores efectos adversos sobre las lesiones. En Colombia, se encontró que pobladores empleaban inicialmente tratamientos «fuertes» (quemaduras, cáusticos) seguidos de «suaves» (plantas) para cicatrizar ${ }^{(11)}$. 
Los pacientes que acuden al Hospital Cayetano Heredia reconocen que la manipulación de sus lesiones con estas sustancias les produce dolor y quemaduras, pero esperan con ello que la lesión cure. En algunos casos tienen éxito, pero la mayoría fracasa, porque la quemadura no es regulada y provoca que muchos tejidos de la lesión (dérmicos y linfáticos) permanezcan con parásitos que posteriormente determinan el crecimiento de la lesión. La termoterapia ha utilizado este conocimiento para producir una quemadura regulada de segundo grado a una temperatura menor a los $52{ }^{\circ} \mathrm{C}$ con un tiempo límite de 30 segundos ${ }^{(17)}$.

En cuanto a las limitaciones, no se consideró el tiempo transcurrido entre la última manipulación y el primer contacto con el centro de referencia, lo cual genera un sesgo de confusión por la cronicidad de la lesión. Además, podría existir el sesgo del entrevistador en obtener adecuadamente la información ya que la población inicialmente tiende a negar la manipulación, subestimando su prevalencia. Otra limitación del estudio fue que no se realizó la tipificación de la especie de Leishmania, por consiguiente, no pudo valorarse si constituye una variable confusora. Futuros estudios con diseño prospectivo y con acceso a métodos moleculares podrían evaluar el efecto de la manipulación de lesiones de LC sobre la carga parasitaria, PCR e identificación de especies de Leishmania.

\section{REFERENCIAS BIBLIOGRÁFICAS}

1. Ampuero J. Leishmaniasis. Módulos técnicos. Series documentos monográficos N8 [Internet]. Lima: Ministerio de Salud. Oficina General de Epidemiología. INS; 2000 [citado el 2 de febrero de 2019]. Disponible en: http://bvs.minsa.gob.pe/local/OGEI/795_MS-OGE106.pdf.

2. World Health Organization. Control of the leishmaniases: report of a meeting of the WHO Expert Committee on the Control of Leishmaniases [Internet]. Geneva, 2010 [citado el 2 de febrero de 2019]. Disponible en: http://whqlibdoc.who.int/trw/WHO_TRS949_eng.pdf.

3. Pineda-Reyes R, Llanos-Cuentas A, Dancuart M. Tratamientos tradicionales utilizados en un área endémica de Leishmaniasis cutánea en el Perú. Rev Perú Med Exp Salud Pública. 2015;32(4):761-765. doi: 10.17843/ rpmesp.2015.324.1770.

4. Mateo S. Situación epidemiológica de la leishmaniosis en el Perú, 2013 (SE 52). Boletín Epidemiológico [Internet]. Lima; 2014 [citado el 2 de febrero de 2019]. Disponible en: https://www.dge.gob.pe/portal/docs/ vigilancia/boletines/2015/09.pdf.

5. Weigel M, Armijos R, Racines R, Zurita C, Izurieta R, Herrera E, et al. Cutaneous leishmaniasis in subtropical Ecuador: popular perceptions, knowledge, and treatment. Bull Pan Am Health Organ. 1994;28(2):142-55.

6. Carrillo-Bonilla L, Trujillo J, Álvarez-Salas L, Vélez-Bernal I. Estudio de los conocimientos, actitudes y prácticas de la leishmaniasis: evidencias del olvido estatal en el Darién Colombiano. Cad Saúde Pública. 2014;30(10):2134-144. doi: 10.1590/0102-311X00139713.

7. Isaza D, Restrepo B, Arboleda M, Casas E, Hinostroza H, Yurgaqui T. La leishmaniasis: conocimientos y prácticas en poblaciones de la costa del Pacífico de Colombia. Pan Am J Public Health. 1999;6(3):177-183.

8. Estrategia Sanitaria de Prevención y Control de las Enfermedades Metaxénicas y otras transmitidas por Vectores. Norma Técnica: Diagnóstico y tratamiento de Leishmaniosis en el Perú [Internet]. Lima: Ministerio de Salud, Perú; 2005 [citado el 2 de febrero de 2019]. Disponible en: http://bvs.minsa.gob.pe/local/MINSA/4520.pdf.
En conclusión, la manipulación de lesiones de leishmaniasis cutánea podría determinar una disminución del resultado del frotis de las lesiones ulcerativas y distorsionar su morfología, lo cual dificulta el diagnóstico clínico. Esto impediría el tratamiento oportuno y gratuito proporcionado por el Ministerio de Salud, que tiene como requisito la demostración parasitológica. El mayor impacto estaría en el primer nivel de atención, donde muchas veces no se cuenta con la experiencia de centros de referencia y se depende principalmente del diagnóstico microscópico. Por ende, es necesario que se implementen programas educativos en áreas endémicas para reducir la manipulación de lesiones de leishmaniasis.

Contribuciones de autoría: JPR, RM, ATZ y ALlC participaron en la concepción del artículo, en el análisis y la interpretación de datos, en la redacción y revisión crítica del manuscrito. JPR, RM y ATZ recolectaron los datos. APR y FA participaron en la evaluación de pacientes, en el registro de la información y en la redacción del manuscrito. Todos los autores aprobaron la versión final y asumen responsabilidad de los contenidos del manuscrito.

Fuentes de financiamiento: Autofinanciado.

Conflictos de interés: Los autores no tienen ningún conflicto de interés que declarar.

9. Mugruza N, Legua P, Llanos E, Maguiña C, Samalvides C. Sensibilidad y especificidad del diagnóstico clínico en Leishmaniasis Cutánea. Medicina [tesis de maestría]. Lima: Facultad de Medicina Alberto Hurtado, Universidad Peruana Cayetano Heredia; 2007.

10. Weigel M, Armijos RR. The traditional and conventional medical treatment of cutaneous leishmaniasis in rural Ecuador. Rev Panam Salud Publica. 2001;10(6):395-404. doi: 10.1590/s1020-49892001001200005.

11. Vasquez ML, Kroeger A, Lipowsky R, Alzate A. Conceptos populares sobre la Leishmaniasis cutánea en Colombia y su aplicabilidad en programas de control. Bol of Saint Panam. 1991;110(5):402-15.

12. Arana BA, Rizzo NR, Navin TR, Klein RE, Kroeger A. Cutaneousleishmaniasis in Guatemala: people's knowledge, concepts and practices. Ann Trop Med \& Parasitol. 2000;94(8):779-86. doi: 10.1080/0003490020012416.

13. Burza S, Croft SL, Boelaert M. Leishmaniasis. The Lancet. 2018;392(10151):951-70. doi: 10.1016/S0140-6736(18)31204-2.

14. Guerra H. Distribution of Leishmaniasis in Peru. En: Walton B, Wijeyaratne P, Modabber F, editores. Research on Control Strategies for the Leishmaniases: Proceedings of an International Workshop held in Ottawa, Canada, 1987. Otawa: IDRC; 1988. p.135-147.

15. Suárez M, Valencia B, Jara M, Alba M, Boggild A, Dujardin J, et al. Quantification of Leishmania (Viannia) Kinetoplast DNA in Ulcers of Cutaneous Leishmaniasis Reveals Inter-site and Inter-sampling Variability in Parasite Load. PLoS Negl Trop Dis. 2015;9(7):1-14. doi: 10.1371/ journal.pntd.0003936.

16. Llanos-Cuentas A, Tulliano G, Araujo-Castillo R, Miranda-Verástegui C, Santamaria-Castrellon G, Ramirez L, et al. Clinical and Parasite Species Risk Factors for Pentavalent Antimonial Treatment Failure in Cutaneous Leishmaniasis in Peru. Clin Infect Dis. 2008;46(2): 223-31. doi: $10.1086 / 524042$.

17. Valencia B, Miller D, Witzig R, Boggild A, Llanos-Cuentas A. Novel LowCost Thermotherapy for Cutaneous Leishmaniasis. PLoS Negl Trop Dis. 2013;7(5):e2196. doi: 10.1371/journal.pntd.0002196. 